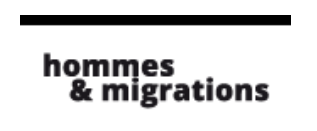

Hommes \& migrations

Revue française de référence sur les dynamiques

migratoires

$1326 \mid 2019$

Londres et ses migrations

\title{
«It Dread Inna Inglan »Une chronique des luttes des Antillais au Royaume-Uni dans les poèmes de Linton Kwesi Johnson
}

\section{David Bousquet}

\section{OpenEdition}

1 Journals

\section{Édition électronique}

URL : https://journals.openedition.org/hommesmigrations/9736

DOI : 10.4000/hommesmigrations.9736

ISSN : 2262-3353

\section{Éditeur}

Musée national de l'histoire de l'immigration

\section{Édition imprimée}

Date de publication : 1 juillet 2019

Pagination : 27-34

ISBN : 978-2-919040-46-9

ISSN : 1142-852X

Référence électronique

David Bousquet, « «It Dread Inna Inglan »Une chronique des luttes des Antillais au Royaume-Uni dans les poèmes de Linton Kwesi Johnson », Hommes \& migrations [En ligne], 1326 | 2019, mis en ligne le 01 janvier 2022, consulté le 16 janvier 2022. URL : http://journals.openedition.org/hommesmigrations/ 9736 ; DOI : https://doi.org/10.4000/hommesmigrations.9736 


\section{«It Dread Inna Inglan»}

Une chronique des luttes des Antillais

au Royaume-Uni dans les poèmes

de Linton Kwesi Johnson

\section{David Bousquet,}

maître de conférences en littérature anglophone à l'université de Bourgogne Franche-Comté.

Linton Kwesi Johnson, dit LKJ, chanteur, poète et musicien de reggae, s'est affirmé depuis les années 1960 comme un artiste noir de premier plan en Angleterre. Né en 1952 en Jamaïque, LKJ arrive à Londres parmi les dizaines de milliers d'immigrants antillais qui s'installent au RoyaumeUni à partir de l'après-guerre. Dans un contexte social tendu, marqué par le racisme et les violences policières auxquels font face les Antillais, LKJ développe une nouvelle forme d'expression artistique entre musique et poésie: la dub poetry. Ce faisant, il devient le porte-voix des luttes des Noirs pour leur reconnaissance au sein de la société britannique.

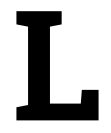

e 21 juin 1948, le navire Empire Windrush aborde les quais de Tilbury, non loin de Londres, avec à son bord plusieurs centaines d'immigrants en provenance de la Caraïbe anglophone qui avaient été embarqués à Kingston, en Jamaïque ${ }^{1}$. L'année précédente, une conférence des pays du Commonwealth avait accordé à chaque pays membre le droit de légiférer individuellement en matière de nationalité et de citoyenneté. En conséquence, le parlement britannique vota une loi créant le statut de «citoyen du Royaume-Uni et des Colonies» qui entra en vigueur le 1er janvier $1949^{2}$. Cette loi octroya de fait la nationalité britannique aux ressortissants des colonies, dont les îles des Antilles anglophones telles que la Jamaïque, la Barbade, Trinité-et-Tobago et Sainte-Lucie. Si les passagers du Windrush avaient anticipé leur départ et furent donc considérés comme illégaux pendant quelques mois, ils furent suivis par de nombreux autres dans les décennies suivantes, avant que la législation ne soit modifiée en $1962^{3}$.
Ainsi, plusieurs dizaines de milliers d'immigrants antillais se sont installés au Royaume-Uni entre la fin de la Seconde Guerre mondiale et le début des années $1960^{4}$. Ils ont participé activement à la

1. Le nombre exact d'immigrants antillais a longtemps fait l'objet de polémiques, mais les archives nationales britanniques établissent à 802 le nombre de passagers ayant déclaré un pays de la Caraïbe comme lieu de résidence.

2. "Citizen of the United Kingdom and Colonies » ou «CUKC » en anglais, statut établi par le British Nationality Act 1948. En 2017 le gouvernement conservateur tenta de revenir sur ce statut, privant de fait des citoyens britanniques d'origine antillaise ayant vécu l'essentiel de leur vie au Royaume-Uni de leur nationalité. Cette réforme, toujours en discussion, est communément appelée le scandale du Windrush.

3. En 1962, le parlement britannique vota le Commonwealth Immigrants Act qui limita fortement l'immigration en provenance des anciennes colonies et restreignit les conditions d'obtention de la nationalité britannique. C'est également la date à laquelle la Jamaïque devint indépendante du Royaume-Uni.

4. On estime que 15000 personnes nées aux Antilles vivaient au Royaume-Uni en 1951, contre 172000 en 1961. 
reconstruction du pays, notamment grâce à des embauches massives dans les chemins de fer et la sécurité sociale, et bénéficiaient en théorie des mêmes droits que les autres citoyens britanniques. Mais la réalité vécue par ces immigrés fut souvent bien différente des promesses faites par le gouvernement et les entreprises publiques ou privées. Même si l'Empire britannique vivait déjà ses derniers soubresauts, l'accueil des immigrés antillais a été marqué par un racisme individuel et institutionnel et de fortes discriminations, notamment dans l'accès au logement. De nombreux incidents opposant communautés blanches et noires émaillent les années 1950. En 1958 ont lieu les émeutes de Notting Hill, un quartier de Londres où résidaient de nombreux Antillais. Elles furent en fait provoquées par à une série d'attaques perpétrées par de jeunes Blancs sur des résidents noirs. En réponse à ces événements, Claudia Jones et d'autres immigrés trinidadiens créèrent le carnaval de Notting Hill, qui existe encore aujourd'hui ${ }^{\mathbf{5}}$.

Ces éléments historiques permettent de brosser à grands traits le contexte dans lequel s'inscrit cet article, qui porte sur les mouvements politiques et les expressions culturelles des Antillais au RoyaumeUni dans les années 1970 et 1980. Un aspect à souligner est le caractère massif de l'immigration antillaise, le Royaume-Uni n'ayant pas connu auparavant de vague d'immigration aussi importante en provenance de ses colonies. Une autre dimension à prendre en compte est que cette immigration fut d'abord parfaitement légale, justifiant de fait les aspirations des immigrants antillais à l'égalité de droit avec les citoyens britanniques, ainsi que leur désir de rester sur le sol du Royaume-Uni de façon permanente.

\section{Le reggae et les musiques populaires jamaïcaines au Royaume-Uni}

La musique occupe une place centrale dans les cultures afro-antillaises, notamment pour des raisons historiques liées à l'esclavage. L'expression musicale est une caractéristique fondamentale des cultures ouest-africaines auxquelles la plupart des esclaves déportés aux Amériques appartenaient. Par ailleurs, la société esclavagiste, en particulier britannique, ne laissait quasiment aucune place à l'expression culturelle des esclaves, et la musique, plus difficile à contrôler que d'autres médiums, en vint à concentrer des fonctions discursives et expressives diverses pour les esclaves et leurs descendants ${ }^{6}$. La Jamaïque est encore aujourd'hui le pays du monde qui compte le plus de musiciens professionnels et de productions phonographiques par habitant. Il n'est donc guère surprenant que nombre d'immigrants en provenance de la Caraïbe anglophone soient des musiciens ${ }^{7}$. La musique, moyen de résistance contre l'ordre colonial et esclavagiste, va remplir une fonction similaire pour lutter contre le racisme et les discriminations dans la société britannique, comme le montre l'histoire du carnaval de Notting Hill.

Dans les années 1950, la musique populaire jamaïcaine connaît des transformations majeures, passant d'un modèle traditionnel ou folklorique à une industrie hypermoderne basée sur un usage pionnier de la technologie musicale. Avec l'émergence des «sound systems» et des studios d'enregistrement, les musiciens et les ingénieurs du son jamaïcains posent les fondations d'une industrie qui va transformer en profondeur la manière de produire et de consommer la musique dans le monde entier ${ }^{8}$. Durant cette période, le lien entre les acteurs musicaux jamaïcains et les communautés antillaises résidant au Royaume-Uni s'avère crucial pour le développement de la musique populaire jamaïcaine. Sans l'apport des artistes et des producteurs britanniques,

5. Rick Blackman relate également l'histoire peu connue d'une campagne menée par des musiciens blancs et noirs en réaction aux émeutes de 1958 : «The Stars Campaign for Interracial Friendship ». Voir Rick Blackman, Forty Miles of Bad Road: The Stars Campaign for Interracial Friendship and the 1958 Notting Hill Riots, Londres, Redwords, 2017.

6. Sur ce point, on pourra notamment se référer aux travaux de Paul Gilroy: There Ain't No Black in the Union Jack, Londres, Hutchinson, 1987 ; The Black Atlantic : Modernity and Double Consciousness, Cambridge, Harvard University Press, 1993.

7. Parmi eux, on peut citer le célèbre chanteur trinidadien de calypso Lord Kitchener, de son vrai nom Aldwyn Roberts, et le Jamaïcain Vincent Ford, qui fonda le premier « sound system » sur le sol britannique dès 1955 sous le nom de Duke Vin The Tickler.

8. Le terme « sound system » se réfère à un ensemble de dispositifs techniques permettant de diffuser du son en public (amplificateurs, enceintes, etc.). En Jamaïque, le terme est utilisé de manière métonymique pour désigner une fête organisée en extérieur où le public est rassemblé pour écouter la musique jouée par le disc-jockey ou DJ sur un « sound system». Les immigrés antillais importèrent ces pratiques au Royaume-Uni, où les « sound systems » sont communément appelés «blues party » ou «blues dance ». Pour des raisons climatiques, ces soirées musicales sont principalement organisées en intérieur, dans des discothèques ou chez des particuliers. L'habitude des Antillais d'écouter de la musique à très fort volume fut l'un des principaux points d'achoppement avec leur voisinage. 
antillais ou non, noirs ou blancs, le reggae et d'autres styles musicaux jamaïcains n'auraient sans doute pas la reconnaissance dont ils bénéficient actuellement. Ainsi, au cours des décennies suivantes, les pratiques des musiciens anglo-jamaïcains vont considérablement influencer le paysage musical britannique et pénétrer profondément la culture populaire9 .

Dans les années 1960, divers styles musicaux jamaïcains vont connaître un succès grandissant en Grande-Bretagne. Il s'agit tout d'abord du ska, puis du rocksteady et enfin du reggae ${ }^{10}$. Dans cette période de formation, la musique jamaïcaine est principalement tournée vers le divertissement des communautés antillaises immigrées. Elle reste cantonnée à des espaces géographiques et symboliques restreints en raison de la discrimination qui s'exerce contre les communautés noires et de politiques visant à criminaliser la musique ${ }^{11}$. La musique noire antillaise, mais également états-unienne, bien que relevant de l'underground, attire toutefois l'attention de certains Britanniques blancs, en particulier les musiciens de la scène rock émergente et les membres de certaines sous-cultures ouvrières, notamment les skinheads. Grâce à ces connexions, la musique jamaïcaine commence à s'ancrer durablement dans la culture populaire britannique ${ }^{12}$.

Dans les années 1970, le reggae évolue vers le style dit «roots» qui atteint une renommée mondiale, notamment en raison de l'incroyable succès de Bob Marley and The Wailers. Ce nouveau style, plus «conscient» que ses prédécesseurs, est marqué par un fort afrocentrisme, notamment sous l'influence du rastafarisme, et jouit d'une grande popularité parmi la deuxième génération d'immigrés antillais au Royaume-Uni ${ }^{13}$. Une scène «roots» britannique va se développer, avec des groupes majeurs tels que Steel Pulse, Aswad, Cimarons ou Misty in Roots, qui connaissent un grand succès au Royaume-Uni, mais également à l'international. Alors que le reggae quitte partiellement l'underground pour rejoindre le mainstream, notamment avec des artistes comme Maxi Priest ou UB40, les musiciens d'origine antillaise vont également tisser des liens de plus en plus étroits avec des artistes de la scène rock. Le mouvement punk, qui émerge au milieu de la décennie, porte un grand intérêt au reggae, pour des raisons de proximité géographique et sociale entre jeunes ouvriers blancs et noirs qui vivent dans les mêmes quartiers et fréquentent les mêmes clubs $^{14}$.

Le reggae continue d'être une musique engagée qui accompagne les luttes des Antillais du
Royaume-Uni, comme en témoignent les émeutes qui se produisent lors du carnaval de Notting Hill en 1976, auxquelles participent d'ailleurs des membres des Clash. L'engagement des musiciens de reggae pour l'amélioration de leur condition sociale et contre le racisme trouve également une illustration frappante dans les concerts géants organisés par l'association Rock Against Racism à la fin de la décennie, en réponse à la montée du National Front et à des déclarations racistes de musiciens pop, notamment Eric Clapton ou David Bowie ${ }^{15}$.

9. Sur la naissance et le développement de la musique populaire jamaïcaine au Royaume-Uni, on pourra se référer au magistral Bass Culture de Lloyd Bradley, journaliste londonien d'origine jamaïcaine, et aux travaux de l'universitaire britannique Dick Hebdige : Lloyd Bradley, Bass Culture: When Reggae Was King, Londres, Penguin, 2001 ; Dick Hebdige, Subculture: The Meaning of Style, Londres/New York, Routledge, 1979 ; Cut'n'Mix : Culture, Identity and Caribbean Music, Londres/New York, Routledge, 1987. Les lecteurs francophones pourront consulter le livre d'Éric Doumerc : Éric Doumerc, Le reggae en Angleterre, 1967-1997, Rosières-en-Haye, Camion Blanc, 2016.

10. Pour les néophytes, le terme « reggae » recouvre souvent l'ensemble de la production musicale jamaïcaine depuis les années 1960. Mais, à strictement parler, le reggae est seulement I'un des sept ou huit genres de musique populaire créés sur lîle dans les dernières décennies. II naît à la fin des années 1960, et est désormais appelé « early reggae » ou « 69 reggae » pour le distinguer du « roots reggae » avec lequel il est souvent confondu. Pour un aperçu plus détaillé de ces divers styles, voir Bradley, op. cit. et Hebdige, op. cit.

11. Ces politiques de criminalisation des expressions musicales trouvent leurs racines dans l'ordre colonial et la répression de toute forme d'expression potentiellement subversive des esclaves. Sur le sujet, voir Bradley, op. cit.

12. Le Royaume-Uni compte depuis les années 1950 d'importantes sous-cultures ouvrières dont l'une des spécificités est un fort attrait pour les musiques noires. Tel est le cas des mods et de la scène "northern soul », mais également des skinheads et du reggae. Un autre indice de l'importance du reggae dans la culture populaire britannique est son utilisation par les fans de football dans les stades. Le Chelsea Football Club, par exemple, utilise le morceau «Liquidator » de Harry J All Stars comme hymne officiel depuis sa sortie en 1969.

13. Le reggae "roots » se caractérise notamment par des rythmes plus lents que les styles précédents et une emphase sur la basse et la batterie, perçues comme des éléments typiquement africains. Du point de vue des paroles, il est marqué par une prédominance des thèmes sociaux et politiques, dits «conscious », alors que les styles précédents se concentraient plutôt sur des thèmes romantiques.

14. Les liens entre reggae et punk sont analysés en détail par Dick Hebdige, op. cit.

15. Sur l'histoire de Rock Against Racism, voir le livre de lan Goodyer, Crisis Music: The Cultural Politics of Rock Against Racism, Manchester, Manchester University Press, 2009. 


\section{Emergence et développement de la «dub poetry»}

Au milieu des années 1970, la reconstruction du Royaume-Uni est quasiment achevée et les chocs pétroliers plongent le pays dans une crise qui va durer presque dix ans. Les immigrés antillais en sont parmi les premières victimes, en raison de la précarité des emplois qu'ils occupent et du racisme à l'embauche qu'ils subissent. Leurs conditions de logement se dégradent encore alors que le chômage augmente. Par ailleurs, l'extrême droite mène un combat acharné contre ces immigrés, symbolisé notamment par le discours sur les "fleuves de sang» prononcé par Enoch Powell quelques années auparavant ${ }^{16}$. Cette offensive se déroule aussi bien au parlement que dans la rue, à la faveur d'attaques racistes commises par des groupes fascistes plus ou moins organisés. Les forces de police, influencées voire infiltrées par l'extrême droite, durcissent leur répression à l'encontre des Antillais britanniques, notamment lors du carnaval de 1976. Une loi de 1824, dite «sus law», leur permet également de contrôler ces populations à loisir sur simple présomption d'une intention de commettre un délit, ce qui détériore très rapidement les relations déjà tendues entre ces citoyens et la police ${ }^{17}$.

C'est dans ce contexte d'effervescence politique et culturelle que naît la «dub poetry». Le terme «dub» renvoie à un sous-genre du reggae inventé par les ingénieurs du son jamaïcains au tournant des années 1970 et qui consiste à remixer intégralement les chansons originales et à leur faire subir un traitement sonore psychédélique ${ }^{18}$. La «dub poetry» se caractérise également par une importance particulière donnée aux paroles et à une volonté de les considérer comme une forme de poésie, donc de littérature «sérieuse». Ainsi, les «dub poets» vont adapter le style vocal des DJ jamaïcains et le formaliser, notamment en le publiant sous forme écrite dans des recueils de poèmes ${ }^{19}$.

Le plus grand nom de la «dub poetry» est sans conteste Linton Kwesi Johnson ou LKJ. Né en Jamaïque en 1952, il rejoint sa mère au Royaume-Uni en 1963 et s'installe dans le quartier de Brixton, dans le Sud de Londres, où résident de nombreux Antillais. Encore lycéen, il devient membre de la branche britannique des Black Panthers et commence ses activités artistiques au sein d'un groupe de percussionnistes et de poètes appelé Rasta Love. En 1978, il publie son premier album, Dread Beat an' Blood, sur lequel il récite ses poèmes sur des accompagnements typiquement «roots reggae». Ce disque marque le début d'une longue collaboration entre Johnson et le musicien Dennis Bovell, lui-même né à la Barbade en 1953 et résidant à Londres, qui deviendra le producteur de tous ses albums. Le poète et le producteur publieront une série d'enregistrements qui marqueront l'histoire du reggae au Royaume-Uni, mais également des communautés antillaises.

Le travail de Johnson est ancré dans les mouvements politiques noirs radicaux des années 1970. Ces derniers sont marqués par une approche basée sur la confrontation directe avec l'État et les groupuscules fascistes, et sont empreints d'idéologies telles que le nationalisme noir, alors très puissant aux États-Unis. Johnson se considère comme le porte-parole de sa communauté et est également reconnu comme tel, aussi bien dans les communautés noires que dans la société britannique dans son ensemble. Au-delà de son travail musical et poétique, il intervient, en effet, régulièrement dans le débat public sur des questions liées à l'immigration, au racisme et aux discriminations.

L'œuvre de Johnson est également notable car elle reflète la volonté des musiciens des années 1970 de faire sortir le reggae de l'underground musical dans lequel il était cantonné et de lui faire accéder à une plus grande légitimité. Dans le cas des «dub

16. Le 20 avril 1968, le député Enoch Powell prononce un discours lors d'une réunion du Parti conservateur à Birmingham dans lequel il dénonce l'immigration de masse, notamment en provenance des pays du Commonwealth. II promet que des fleuves de sang ("rivers of blood») inonderont le pays si l'immigration ne cesse pas et évoque les nuisances sonores causées par les immigrés antillais.

17. Le Vagrancy Act de 1824 était à l'origine destiné aux vagabonds et permettait de les arrêter sur simple suspicion, d'où le terme argotique de «sus law». La loi fut abolie en 1981 suite aux émeutes de Brixton.

18. Le dub est l'une des innovations majeures des ingénieurs du son jamaïcains, tels que King Tubby et Lee Scratch Perry, qui a permis le développement de la musique électronique grâce à l'invention de l'échantillonnage et du remix. Voir Bradley, op. cit. et Hebdige, op. cit. pour plus de précisions.

19. Bien que Johnson en soit le fondateur, la « dub poetry » est pratiquée par de nombreux autres artistes dans le monde depuis les années 1980. Parmi les plus grands « dub poets », on pourra citer Mutabaruka et Mikey Smith (Jamaïque), Lillian Allen (Canada) et Benjamin Zephaniah (Royaume-Uni). Tous participent de la volonté de faire passer la tradition orale des DJ jamaïcains à la sphère littéraire. Pour une analyse plus poussée du genre, voir Carolyn Cooper, Noises in the Blood: Orality, Gender and the "Vulgar" Body of Jamaican Popular Culture, Londres/ Basingstoke, Macmillan Caribbean, 1993 ; Christian Habekost, Verbal Riddim: The Politics and Aesthetics of African-Caribbean Dub Poetry, Amsterdam/Atlanta, Rodopi B.V., 1993. 
poets», cette aspiration passe par une reconnaissance littéraire et universitaire. Ils publient ainsi leurs textes sous forme écrite, contrairement aux DJ, chez des éditeurs reconnus. Johnson fut par exemple le deuxième poète vivant à voir une anthologie de son travail publiée dans la collection Penguin Classics en 2002. Il a également reçu de nombreux prix littéraires et entretient des liens forts avec le monde universitaire. Outre les nombreuses lectures de poésie qu'il donne dans des universités, il s'est vu attribuer, parmi d'autres distinctions, le titre de docteur honoris causa par de prestigieuses institutions britanniques ${ }^{20}$.

Un dernier aspect à souligner dans le travail poétique de Johnson est son emploi du créole jamaïcain comme médium linguistique quasi exclusif. Si

20. Pour plus d'informations sur la biographie, la bibliographie et la discographie de Johnson, voir son site internet : http://www. lintonkwesijohnson.com/. 
l'idée d'écrire et de publier des textes littéraires en créole avait été développée dès les années 1940 par Louise Bennett, plus connue sous son nom de scène, Miss Lou, puis par Edward Kamau Brathwaite dans les années 1960, les «dub poets», dont Johnson, sont les premiers à utiliser uniquement cette variété de langue et donc à exclure l'anglais standard. Ils inversent ainsi les hiérarchies coloniales et la diglossie qui construit le créole comme variété de langue inférieure au standard, souvent considérée comme déviante ou défaillante. Il s'agit également pour les «dub poets» de réhabiliter les cultures afro-antillaises dans leur ensemble et de conférer au créole une reconnaissance en tant que médium légitime pour l'expression linguistique et littéraire des Antillais ${ }^{21}$.

\section{Chronique des luttes politiques des Antillais britanniques}

Malgré son intérêt pour le rastafarisme, Johnson se situe avant tout dans une perspective marxiste relativement orthodoxe. Comme le suggère l'anthologie de ses poèmes publiée chez Penguin, son œuvre est conçue principalement comme une chronique des tribulations des Antillais du Royaume-Uni. L'anthologie est organisée de manière chronologique, avec une section par décennie allant des années 1970 aux années 1990. Tous les poèmes, sauf deux, traitent explicitement de sujets politiques et s'ancrent dans la réalité sociale des Antillais du Royaume-Uni, notamment à travers un appareil de paratexte et de notes de bas de page offrant des précisions historiques et contextuelles ${ }^{22}$.

Dans la section consacrée aux années 1970, on trouve, tout d'abord, des poèmes qui dénoncent en termes généraux le racisme, la discrimination à l'embauche et au logement, et la pauvreté imposée par un système de domination postcolonial. C'est le cas, par exemple, du poème «Inglan is a Bitch», au titre pour le moins provocateur. Le refrain insiste sur l'impossibilité pour les immigrés d'échapper à l'Angleterre et aux discriminations qui leur sont imposées. Les couplets décrivent le parcours typique d'un immigré antillais qui cherche du travail et ne trouve que des emplois subalternes et mal payés. Le protagoniste du poème est d'abord embauché sur les chantiers du métro londonien, puis fait la plonge dans un hôtel et travaille dans la construction des infrastructures routières. Il finit par trouver un emploi dans une usine pendant quinze ans avant de se faire licencier et de se retrouver au chômage à l'âge de 55 ans.
Dans la même veine, «Want fi Goh Rave» met en scène des rencontres entre le narrateur du poème et de jeunes Antillais qu'il croise dans la rue. Ils parlent tous de leur condition misérable, évoquant l'impossibilité de trouver un logement ou un emploi, et la nécessité de recourir à des activités criminelles pour survivre. "Sonny's Lettah», l'un des poèmes les plus connus de Johnson, se présente comme une lettre envoyée par un jeune immigré à sa mère restée en Jamaïque. Le poème raconte de manière poignante l'agression subie par le narrateur Sonny et son cousin Jim lors d'un contrôle de police. Dans un geste d'autodéfense, Sonny fait tomber un policier qui se cogne la tête et décède. La lettre est écrite depuis la prison de Brixton où le narrateur est incarcéré en attendant son jugement.

Ces poèmes font référence à la condition sociale des Antillais par le biais de situations fictionnelles, bien que crédibles et réalistes. Mais on trouve également dans cette section des poèmes qui se réfèrent à des événements historiques précis et documentés. «Forces of Victory», par exemple, est dédié au collectif antiraciste Race Today, dont Johnson a fait partie, et aux organisateurs du carnaval de Notting Hill. Le poème décrit la mobilisation des Antillais lors du carnaval de 1978 qui fait suite aux incidents de 1976. «It Dread Inna Inglan» est dédié à George Lindo, un ouvrier de Bradford injustement accusé d'avoir commis un braquage, et décrit les manifestations qui eurent lieu pour sa libération. On retrouve le même type de dispositif dans le poème «Reggae fi Peach»

21. Durant la période coloniale, les esclaves développent des parlers qui emploient les bases lexicales des langues européennes des maîtres mais les transforment considérablement, notamment par l'utilisation de caractéristiques syntaxiques et phonologiques issues des langues ouest-africaines. S'instaure ainsi une forte diglossie entre les variétés standards de l'anglais et les créoles, souvent assimilés à du mauvais anglais («broken English »). Les créoles sont également considérés comme inaptes à toute forme d'expression littéraire et poétique, notamment car ce sont des variétés principalement orales. L'un des enjeux de la période qui précède et qui suit les indépendances sera donc d'imposer l'idée qu'une littérature en créole est possible et tout aussi « sérieuse » que la littérature écrite en anglais standard, et donc d'écrire en créole. Voir Carolyn Cooper, op. cit. et Christian Habekost, op. cit. pour plus de précisions.

22. Tous les poèmes de Johnson mentionnés ici, sauf un, sont issus de son anthologie intitulée Mi Revalushanery Fren: Selected Poems, Londres, Penguin, 2002. La plus grande partie a également été publiée sous forme d'enregistrements audio, avec ou sans accompagnement musical, sur les albums de Johnson. «Reggae fi Peach », non publié dans l'anthologie, est disponible sur l'album Bass Culture, Island Records, 1980. 
qui est dédié à la mémoire de Blair Peach, un militant antifasciste néo-zélandais décédé des mains de la police lors d'une manifestation de l'Anti-Nazi League en avril 1979.

\section{La grande insurrection}

Cette chronique des luttes des Antillais britanniques se poursuit dans les années 1980, notamment avec deux poèmes qui traitent des événements ayant mené aux émeutes urbaines qui ont agité le pays durant l'été 1981. «New Cross Massakah» rend hommage aux victimes de l'incendie de New Cross, parfois également appelé incendie de Deptford. Le 18 janvier 1981, un groupe d'adolescents est rassemblé dans un appartement de New Cross Road, dans le Sud-Est de Londres, pour fêter l'anniversaire d'Yvonne Ruddock et d'Angela Jackson, toutes deux d'origine antillaise. Sans que l'on sache précisément pourquoi à ce jour, un incendie éclate et treize jeunes trouvent la mort, dont Yvonne Ruddock, et vingt-six sont grièvement blessés. Une quatorzième victime se donne la mort un an après les faits.

Une enquête est menée et suit diverses pistes, notamment celle d'une attaque raciste puisque des groupuscules du National Front étaient actifs dans le quartier. Un autre motif possible aurait été une tentative de faire cesser les nuisances sonores, des voisins s'étant plaints du bruit durant la nuit. Un premier procès se tient dans les mois qui suivent et un non-lieu est prononcé. Les familles des victimes contestent ce verdict, dénonçant notamment l'enquête policière qui met en avant la thèse improbable d'un accident. Un appel est lancé devant la Haute Cour de Justice, qui met en évidence les carences de l'enquête mais confirme le non-lieu. La Haute Cour rouvre une enquête en 2002, qui aboutira à nouveau à un non-lieu faute de preuves en 2004. Johnson, quant à lui, se prononce clairement en faveur de la thèse d'une attaque raciste dans une note de bas de page de son poème.

Cette tragédie va mener à une forte mobilisation des Antillais britanniques pour réclamer justice mais aussi pour que cessent les violences racistes, souvent couvertes et parfois même commises par la police. Dès le 25 janvier, un comité d'action est créé et se réunit dans un club du quartier de New Cross. La réunion se termine par une manifestation de plus de 1000 personnes qui se rendent sur les lieux du drame et bloquent la circulation pendant plusieurs heures. Le comité organise ensuite une grande marche des
Noirs le 2 mars qui réunit plus de 20000 personnes ${ }^{23}$. Dans ce contexte, un incident impliquant un jeune Noir et la police à Brixton en avril dégénère en trois jours d'émeutes qui opposent les résidents du quartier, majoritairement antillais, à la police. Plus de 300 personnes sont blessées et plus de 80 arrêtées, plus de 100 véhicules sont détruits et plus de 150 bâtiments endommagés ou brûlés. Johnson célèbre ces émeutes dans le poème «Di Great Insohreckshan » et les interprète comme une réaction justifiée face à une oppression intolérable subie par les Noirs.

\section{(र Cette chronique des luttes des Antillais britanniques se poursuit dans les années 1980, notamment avec deux poèmes qui traitent des événements ayant mené aux émeutes urbaines qui ont agité le pays durant l'été 1981.}

À la suite des émeutes de Brixton, de nombreux autres quartiers populaires de grandes villes britanniques vont s'enflammer pendant l'été 1981 lors d'affrontements entre la police et les communautés noires, notamment Southall à Londres, Moss Side à Manchester, Toxteth à Liverpool, Handsworth à Birmingham et Hyson Green à Nottingham. Ces événements mèneront à la publication du rapport Scarman en novembre 1981, qui met clairement en évidence le racisme institutionnel de la police britannique et les abus d'autorité caractérisés, favorisés par les «sus laws ${ }^{24} \gg$. Ce rapport transformera en profondeur les relations entre la police et les citoyens britanniques noirs, même si une forme de racisme institutionnel demeure, comme le révèle la mort de Stephen Lawrence en $1993^{25}$. Le combat des Antillais contre le racisme et

23. «Black People's Day of Action», également appelé «Black March».

24. Suite aux émeutes, le gouvernement commissionne une enquête confiée à Lord Scarman pour établir les causes des violences et formuler des recommandations. Le rapport a été publié sous forme de livre en 1982.

25. Stephen Lawrence était un adolescent noir qui trouva la mort lors d'une attaque raciste perpétrée par un groupe de jeunes Blancs alors qu'il attendait le bus. Les suspects furent arrêtés par la police mais aucune charge ne fut retenue contre eux, soi-disant faute de preuve. II fallut attendre 2011 pour que deux des agresseurs soient enfin condamnés, et l'affaire demeure l'une des plus emblématiques du racisme qui mine les forces de police britanniques. 
la discrimination continue, comme l'atteste le poème «Liesense fi Kill» de 1998 où Johnson énumère les noms de citoyens noirs morts aux mains de la police dans des circonstances suspectes au cours des trois décennies que couvre son anthologie. Le poème fait mention d'une douzaine de victimes et met directement en cause la police et les gouvernements successifs dans leur décès.

Pour clore ce tour d'horizon des poèmes de Johnson, il convient de signaler que plusieurs d'entre eux sont spécifiquement dédiés à la musique et au rôle qu'elle joue dans la vie culturelle des Antillais britanniques, mais aussi dans leurs luttes pour l'égalité et la justice. C'est le cas par exemple de «Street 66 », dans lequel Johnson décrit une scène habituelle durant laquelle la police interrompt violemment une «blues dance», une soirée musicale organisée chez un particulier $^{26}$. Dans ce poème, comme dans «Bass Culture», «Dread Beat an' Blood » ou «Five Nights of Bleeding», Johnson met en avant le caractère cathartique et libératoire de la musique qui est caractéristique des cultures afro-antillaises marquées par l'esclavage. Mais ces poèmes mettent également en lumière un lien fort entre la musique et la violence, le plaisir et l'excitation suscités par la musique étant aisément réversibles dans un déchaînement de violence entre les membres de la communauté eux-mêmes, ou entre la communauté et les forces de l'ordre.

\section{Conclusion}

La pochette de l'album Dread Beat an' Blood montre une photographie sur laquelle on voit Johnson, mégaphone à la main, devant un poste de police. Il fait face à une foule rassemblée pour manifester, et derrière lui se tiennent deux policiers qui gardent l'entrée du bâtiment. Cette image illustre clairement l'engagement de Johnson dans les luttes qu'il mentionne dans ses poèmes. Tel est le cas de nombreux autres musiciens et artistes antillais, qui furent non seulement des témoins directs mais également des acteurs des événements mentionnés dans cet article ${ }^{27}$. Il n'est guère surprenant que la musique, composante fondamentale de la vie sociale dans les cultures afro-antillaises, ait été la cible directe de politiques racistes dans le contexte britannique, ni qu'en retour les musiciens se soient mobilisés pour défendre leur droit à exercer leur art et, au-delà, les droits des Antillais du Royaume-Uni à la liberté d'expression et à l'égalité de traitement avec les autres citoyens britanniques. En ce sens, et comme le montre l'exemple de Johnson, dire la lutte, c'est également vivre la lutte.

26. Une scène similaire est décrite dans le morceau «Blues Dance Raid » de Steel Pulse.

27. Pour ne citer qu'un seul exemple, Clarence Baker, membre de Misty in Roots, resta cinq mois dans le coma après avoir été molesté par la police lors de la manifestation de l'Anti-Nazi League dans laquelle Blair Peach trouva la mort en 1979. 